\section{Die Bedeutung der gesundheitsbezogenen Lebensqualität für die Bewertung interventioneller Maßnahmen bei Patienten mit COPD}

Zusammenfassung: Die vorliegende Übersicht bietet einen kritischen Überblick über Methoden und Ergebnisse von Studien zur Effektivität der Rehabilitation bei chronisch-obstruktiven Lungenerkrankungen (COPD) hinsichtlich der gesundheitsbezogenen Lebensqualität (HRQOL). In 14 (45\%) der 31 analysierten Studien wurde ein krankheitsspezifisches und in 12 (39\%) Studien ein krankheitsübergreifendes (generelles) Verfahren zur Messung der HRQOL verwendet. In weiteren 4 der verbleibenden 5 Studien wurde ein generelles und ein krankheitsspezifisches Verfahren eingesetzt. Die generellen Verfahren erwiesen sich als nicht weniger sensitiv für therapieinduzierte Veränderungen im Vergleich zu der krankheitsspezifischen Methodik. Die Analyse der publizierten Studien legt eine kombinierte Verwendung von generellen und spezifischen Verfahren nahe. In 12 (39\%) von 31 Studien zu den Auswirkungen von Rehabilitationsmaßnahmen auf die HRQOL ergaben sich anhaltend positive Effekte einzelner Interventionen wie der operativen Lungenvolumenreduktion, dem Einsatz von Pharmaka, dem Training der Atemmuskulatur und psychologischen Interventionen sowie integrierten Rehabilitationsprogrammen. Stationäre und ambulante Programme unterscheiden sich in der Dauerhaftigkeit ihrer Wirkung. In 2 von 3 Studien zu einem stationären Rehabilitationsprogramm trat nach anfänglicher Verbesserung zum Katamnesezeitpunkt eine Verschlechterung der HRQOL ein. Andererseits hielt die Wirkung von 3 der 4 im ambulanten oder häuslichen Kontext durchgeführten Programme bis zur Katamnese an.

The Significance of Health Related Quality of Life for the Evaluation of Interventional Measures in Patients with COPD: Health related quality of life (HRQOL) is an important criterion for the evaluation of rehabilitation measures in patients with chronic obstructive pulmonary disease (COPD). The present paper reviews the current literature about the effects of pulmonary rehabilitation on the HRQOL of patients with COPD. The aim is to summarize critically methods, results and unanswered issues of the present research on the effects of pulmonary rehabilitation on HRQOL. The rehabilitation of patients suffering of COPD is mainly based on six types of interventions: 1. long-term oxygen therapy (LTO), 2. pharmacological management, 3. surgical therapy (bilateral reduction of lung volume), 4. physical therapy, 5. nutritional therapy (special diets), and 6. psycho-social interventions (e.g. psychotherapy, training

Pneumologie 2001; 55: $91-99$

(c) Georg Thieme Verlag Stuttgart · New York ISSN 0934-8387

\author{
G. Würtemberger ${ }^{1}$, B. O. Hütter ${ }^{2}$ \\ ${ }^{1}$ Abteilung für Innere Medizin, Pneumologie; Reha-Zentrum \\ Reichshof; Reichshof-Eckenhagen \\ (Leiter: PD Dr. G. Würtemberger) \\ 2 Neurochirurgische Klinik, Universitätsklinikum der Rheinisch- \\ Westfälischen Technischen Hochschule (RWTH); Aachen \\ (Leiter: Prof. Dr. J. M. Gilsbach)
}

and education). Thirty-one studies could be included in which HRQOL served as an outcome criterion for the rehabilitation of COPD patients. In 14 (45\%) studies exclusively a disease-specific measure for the assessment of HRQOL was employed, while in $12(39 \%)$ studies a generic instrument was applied. In the remaining five (16\%) studies two ore more measures were used, whereas four of them combined a generic and a disease-specific method. The St. Georges Respiratory Questionnnaire (SGRQ) und the Chronic Respiratory Disease Questionnaire (CRDQ) belonged to the group of the specific instruments, while among the generic measures the Sickness Impact Profile (SIP), the Nottingham Health Profile (NHP), the SF-36 and the Quality of Well-Being Scale (QWB) were most frequently used in COPD patients. The surgical bilateral reduction of lung volume, pharmacological therapy, upper extremities muscle training and psychological measures as single interventions proved to have persistent positive effects on the HRQOL. Several rehabilitation programs, composed of a wide variety of different interventions were effective in terms of HRQOL. On the other hand, at follow-up, the short-term positive effects had decreased in two of the three studies, where the rehabilitation took place exclusively in an inpatient setting. However, in three of four programs implemented in an outpatient setting, a persistent positive effect on HRQOL could be demonstrated. In conclusion from the as of yet available findings, we suggest for future studies to use only such measures of HRQOL which have been tested psychometrically in patients with COPD and to combine disease-specific and generic measures. In order to achieve lasting positive effects of rehabilitation on HRQOL, outpatient settings or ambulatory refreshment sessions following rehabilitation on an inpatient basis should be preferred.

\section{Einleitung}

Trotz der Fortschritte in der Behandlung von chronisch obstruktiven Lungenerkrankungen (COPD) beträgt die 5-Jahres-Überlebensrate von hypoxämischen Patienten mit COPD bei maximaler medizinischer Therapie etwa 30\% [1-3]. Hat die Erkrankung ein bestimmtes Stadium erreicht, ist eine Heilung nicht mehr möglich und der Verlust der Lungenfunktion schreitet unumkehrbar fort [3,4]. Daher konzentriert sich die Behandlung von Patienten mit COPD und Hypoxämie in erster Linie auf die Linderung der Krankheitssymptome, insbesondere von Atemnot (Dyspnoe), die Verringerung der Notwendigkeit von Krankenhausaufenthalten und die Verbesserung bzw. Stabilisierung der gesundheitsbezogenen Lebensqualität (HRQOL) [5,6]. Vor diesem Hintergrund ist die 
HRQOL ein bedeutsames Kriterium zur Bewertung der Effektivität pneumologischer Rehabilitationsmaßnahmen bei hypoxämischen Patienten mit COPD.

\section{Grundlagen der pneumologischen Rehabilitation von Patienten mit COPD}

In einer modernen Definition pneumologischer Rehabilitation wurde 1994 auf dem Workshop des National Institute of Health ein multidimensionaler, interdisziplinärer Ansatz mit dem Ziel, ein Maximum an Unabhängigkeit und Leistungsfähigkeit der Patienten zu erreichen und zu erhalten, gefordert [7]. Die Rehabilitation and Chronic Care Scientific Group der Europäischen Gesellschaft für Lungenkrankheiten postulierte die folgenden, in Tab.1 aufgelisteten Ziele pneumologischer Rehabilitationsmaßnahmen [8].

\section{Tab. 1 Ziele der pneumologischen Rehabilitation}

1. Evaluierung und Monitoring von Behandlungen

2. Linderung der leistungsmindernden physischen und psychischen Symptome

3. Wiederherstellung der bestmöglichen funktionellen Leistungsfähigkeit

4. Förderung der sozialen Reintegration

Die Rehabilitation von hypoxämischen Patienten mit COPD beruht im Wesentlichen auf den Säulen:

1. physikalische Therapie;

2. psychosoziale Maßnahmen einschließlich Patientenschulung und -training;

3. medikamentöse Therapie und

4. Ernährungstherapie [9].

Das Spektrum der psychosozialen Interventionen wiederum umfasst acht Bereiche:

1. Psychotherapie und psychologische Beratung;

2. Patientenschulung;

3. Patiententraining (z.B. Stressimpfungstraining);

4. Entspannungstherapie;

5. Verhaltenstraining;

6. Beeinflussung von Krankheitswahrnehmung und Krankheitsverarbeitung;

7. Angehörigenberatung und

8. Interventionen zur Verbesserung der Compliance [10].

Die Beurteilung der Wirksamkeit pneumologischer Rehabilitationsmaßnahmen erfordert in einem Mehr-Ebenen-Ansatz die Berücksichtigung unterschiedlicher Bereiche, die zusammen erst den Gesamtzustand eines Patienten angemessen abbilden. Hierzu gehören:

1. der körperliche Zustand einschließlich der Lungenfunktion;

2. die Schwere der Dyspnoe;

3. die Anstrengungstoleranz;

4. die HRQOL und

5. die psychologische Befindlichkeit.

Hierbei besteht das Oberziel der pneumologischen Rehabilitation in einer anhaltenden Verbesserung, zumindest aber einer
Stabilisierung der HRQOL [2,7,9]. Jedoch besteht neben Anwendungsfragen in der klinischen Routine immer noch ein erheblicher methodischer Forschungsbedarf [5]. Einen umfassenden Überblick über den bisherigen Forschungsstand bietet die Metaanalyse von Lacasse u. Mitarb. [11], in der die Ergebnisse von 14 Studien zur pneumologischen Rehabilitation von Patienten mit COPD zusammenfassend ausgewertet wurden. In methodologischer Hinsicht fällt auf, dass nur fünf Operationalisierungen von Lebensqualität in konzeptueller wie methodischer Hinsicht den Anforderungen an ein Verfahren zur Erfassung von HRQOL entsprechen [11]. Das Ziel der vorliegenden Arbeit besteht daher in einer kritischen Übersicht über die bisher publizierten Studien, in denen die HRQOL zur Beurteilung der Effektivität pneumologischer Rehabilitationsmaßnahmen erhoben wurde.

\section{Kriterien für den Einschluss der referierten Studien}

Um angesichts der bisher noch begrenzten Forschungserfahrungen die Perspektive nicht von vornherein zu sehr einzuengen, wurde im Gegensatz zu der Metaanalyse von Lacasse u. Mitarb. [11] darauf verzichtet, nur randomisierte Studien zu berücksichtigen. Das wichtigste Einschlusskriterium fordert vielmehr die Verwendung eines publizierten Verfahrens zur standarisierten Messung der HRQOL. Da gemischte Studienstichproben mit anderen Diagnosegruppen wie Asthma nicht sinnvoll sind [2], wurden Studien, die auf heterogenen Stichproben beruhen, nur dann berücksichtigt, wenn eine getrennte Auswertung für die Patienten mit COPD vorlag.

\section{Ergebnisse}

\section{Untersuchungen zur Wirksamkeit pharmakologischer Interventionen}

Van Schayck u. Mitarb. [12] untersuchten die Wirkung von Beclomethason-Dipropionat (BDP) auf die HRQOL von 28 Patienten mit COPD. Zur Erfassung der HRQOL diente das Nottingham Health Profile (NHP) [13]. Obwohl durch BDP die Lungenfunktion $\left(\mathrm{FEV}_{1}\right)$ signifikant gesteigert wurde, fand sich in keiner der sechs Subskalen des NHP eine statistisch bedeutsame Verbesserung [12]. Jones und Bosh [14] berichteten über Ergebnisse einer Längsschnittstudie, in der sie den Effekt einer Plazebobehandlung mit der Wirkung einer Tagesdosis von 50 bzw. $100 \mu \mathrm{g}$ Salmeterol auf die HRQOL von 283 Patienten mit COPD untersuchten. Es wurden zum einen das krankheitsspezifische St. Georges Respiratory Questionnaire (SGRQ) [15] und zum anderen der SF-36 [16] zur Erfassung der krankheitsübergreifenden HRQOL eingesetzt. Das Studiendesign entsprach einer randomisierten, plazebokontrollierten Doppel-Blind-Studie. Die HRQOL wurde bei den Patienten zu Beginn der Studie und 16 Wochen nach der Behandlung erhoben. Im Vergleich zur Voruntersuchung ver-

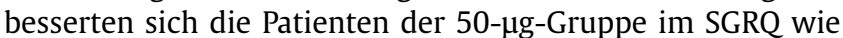
auch im SF-36 substanziell. Diese Veränderungen waren signifikant größer im Vergleich zu den Patienten, die $100 \mu \mathrm{g}$ Salmeterol erhalten hatten. In der SF-36 Subskala „Emotionale Rollenfunktion“ fand sich in der hoch dosierten Gruppe sogar eine signifikante Verschlechterung der HRQOL. Dieser Effekt wurde von den Autoren durch unerwünschte psychotrope Nebenwirkungen von Salmeterol in der hohen Dosierung erklärt, die sich beeinträchtigend auf die HRQOL auswirkten 
[14]. DiLorenzo und Mitarb. [17] verglichen die Wirksamkeit von Salmeterol und von Theophyllin während einer 3-monatigen Periode auf die HRQOL von 178 Patienten mittels des SF36. Beide Pharmaka führten zu einer signifikanten Verbesserung der HRQOL, wobei jedoch die Patienten, die Salmeterol erhalten hatten, in den Bereichen des physischen Funktionierens $(p=0,02)$, in der sozialen Rollenfunktion $(p=0,004)$ und in der Gesundheitswahrnehmung $(p=0,03)$ jeweils mehr profitiert hatten, als diejenigen, die mit Theophyllin behandelt worden waren [17].

\section{Studien zur Auswirkung der chirurgischen Verminderung des Lungenvolumens auf die HRQOL}

In jüngerer Zeit wird zunehmend eine bilaterale chirurgische Verkleinerung des Lungenvolumens bei Patienten mit schwerer COPD durchgeführt, um hierdurch die Lungenfunktion $\mathrm{zu}$ verbessern und die Einschränkungen durch Dyspnoe zu verringern. Cordova u. Mitarb. [18] überprüften die Dauerhaftigkeit der Verbesserung der HRQOL nach bilateraler Lungenvolumenreduktion bei 26 Patienten, die vor dem Eingriff und 3, 6, 12 und 18 Monate danach mittels des Sickness Impact Profiles (SIP) [19] hinsichtlich ihrer HRQOL untersucht worden waren. Es zeigte sich eine hoch signifikante Verbesserung der HRQOL in allen SIP Summenscores zwischen der Voruntersuchung und der ersten Nachuntersuchung drei Monate später, die bei den folgenden Katamneseuntersuchungen bis zur Spätkatamnese 18 Monate später stabil blieb [18]. Jedoch standen in der Studie von Cordova u. Mitarb. [18] aufgrund eines erheblichen Drop-outs zur Spätkatamnese nur die Daten von 6 Patienten zur Verfügung.

Die Studie von Moy u. Mitarb. [20] weist die Besonderheit auf, dass die HRQOL zunächst nach einer bilateralen Lungenverkleinerung und dann nach Abschluss der Rehabilitation erhoben worden war. Leider wurden die Bestandteile des Rehabilitationsprogramms nicht im einzelnen beschrieben. Moy u. Mitarb. [20] fanden einen differenziellen Effekt der Rehabilitation auf die soziale Rollenfunktion, die mittels des SF-36 erhoben worden war, während die Operation insbesondere zu Verbesserungen in den Bereichen des physischen und sozialen Funktionierens und der Vitalität (SF-36 Subskalen) führte. O'Brien u. Mitarb. [21] untersuchten neben einer Reihe von anderen Variablen die Auswirkungen einer bilateralen Lungenvolumenreduktion auch hinsichtlich der HRQOL, die mittels des SIP erfasst wurde. Im SIP Total Score zeigten sich bei 9 Patienten mit einem $\mathrm{PaCO}_{2}>45 \mathrm{mmHg}$ und in der Gruppe von 19 Patienten mit einem $\mathrm{PaCO}_{2}<45 \mathrm{~mm} \mathrm{Hg}$ zur Nachuntersuchung 3 bis 6 Monate nach der Operation jeweils deutliche $(p<0,001)$ Verbesserungen der HRQOL [21].

\section{Die Effekte von Langzeitsauerstofftherapie (LTO) auf die HRQOL}

Die Wirksamkeit von Langzeitsauerstofftherapie (LTO) hinsichtlich einer signifikanten Verlängerung der Überlebenszeit kann mittlerweile als hinreichend belegt angesehen werden $[1,3,22]$. Aus der NOTT-Studie folgte, dass eine kontinuierliche Sauerstoffgabe über mindestens $18 \mathrm{Std}$. pro Tag die Überlebensrate der Patienten verdoppelte [22]. Auch wenn in mehreren Studien gezeigt werden konnte, dass die Einführung von LTO zu einer erhöhten Mobilität, gesteigerter Kapazität im Alltagsleben und verbessertem Schlaf führte
$[23,24]$, so fehlen bislang Belege für einen andauernden positiven Effekt von LTO auf die HRQOL.

In einer Längsschnittstudie zum Effekt von LTO auf die HRQOL von 150 Patienten mit COPD fand sich 12 Monate nach Beginn der Therapie kein signifikanter Unterschied im Sickness Impact Profile (SIP) im Vergleich zu einer Kontrollgruppe [25]. Crockett u. Mitarb. [26] untersuchten in einer prospektiven Längsschnittstudie 114 Patienten nach Einführung von LTO hinsichtlich ihrer HRQOL 3, 6 und 12 Monate später mittels des Nottingham Health Profiles (NHP), des SF-36 und des Chronic Respiratory Disease Questionnaire (CRDQ) [27]. Im Vergleich zwischen der HRQOL vor und nach der Einführung von LTO zeigten sich signifikante kurzfristige Verbesserungen, die jedoch zum Zeitpunkt der Spätkatamnese nach 12 Monaten nur noch teilweise nachweisbar waren. Hierbei erwies sich das Geschlecht der Patienten als wichtige ModeratorVariable [26].

\section{Die Effekte von Flüssigsauerstofftherapie (LOX) auf die gesundheitsbezogene Lebensqualität (HRQOL)}

Durch den Wechsel von Sauerstoffkonzentratoren zur Flüssigsauerstofftherapie (LOX) gewinnen hypoxämische Patienten mit COPD potenziell mehr Mobilität, weil die Sauerstoffquelle tragbar ist $[2,28]$. Daher wurde LOX auch deshalb eingeführt, um durch die Erhöhung der Mobilität die HRQOL der betroffenen Patienten zu verbessern. Jedoch liegen bisher nur wenige Studien zur HRQOL von COPD-Patienten unter LOX-Therapie vor. So verwendeten Vergeret u. Mitarb. [29] nur einen adhoc-Fragebogen zur Erfassung der Alltagsaktivitäten, der weder den konzeptuellen noch den methodischen Anforderungen des Konzepts der HRQOL entspricht.

\section{Die Wirksamkeit von einzelnen Interventionen in der Rehabilitation von Patienten mit COPD}

Die wichtigsten Ziele der pneumologischen Rehabilitation von Patienten mit COPD bestehen darin, die Symptome zu lindern und die Möglichkeiten der Patienten im Alltagsleben $\mathrm{zu}$ verbessern [30]. Eine physiologisch orientierte Intervention basiert auf der Annahme, dass bei COPD-Patienten die Überanstrengung der Atemmuskulatur die Dyspnoe verschlimmert. Daher soll durch eine maschinengestützte Beatmung während mehrerer täglicher Intervalle eine Entlastung und Kräftigung der Atemmuskulatur erreicht werden. In einer methodisch gut kontrollierten Studie von Martin [31] fand sich jedoch keine Verbesserung in der Anstrengungstoleranz, Blutgasen, Atemnot oder der HRQOL (CRDQ) im Vergleich zu einer Gruppe, die eine Scheinbehandlung erhalten hatte [31]. Ein gezieltes Training der Atemmuskulatur, insbesondere solcher Muskeln, die die Einatmung unterstützen, bildet eine weitere Maßnahme in der pneumologischen Rehabilitation von Patienten mit COPD. Guyatt u. Mitarb. [32] fanden in einer Studie zur Wirksamkeit von Übungen zum Training der Atemmuskulatur keinen positiven Effekt auf die HRQOL, die mittels des CRDQ erhoben worden war.

Strijbos u. Mitarb. [33] beobachteten eine signifikante Verbesserung der HRQOL (CRDQ) bei 30 Patienten mit COPD, die neben einem Aerobic-Training noch eine Unterweisung in Entspannungstechniken und Atemgymnastik mit gesteuerter Inspiration und dosierter Lippenbremse erhalten hatten. Von 
Tab. 2 Studien zur Effektivität von integrierten Rehabilitationsprogrammen auf die HRQOL von COPD-Patienten

\begin{tabular}{|c|c|c|c|c|c|c|}
\hline Autoren & Methode HRQOL & $\begin{array}{l}\text { Interventions- } \\
\text { gruppe (n) }\end{array}$ & Kontrollgruppe & Längsschnitt & randomisiert & $\begin{array}{l}\text { Katamnese } \\
\text { (Abstand) }{ }^{\#}\end{array}$ \\
\hline Emery et al., 1991 & QWB & 64 & nein & ja & - & keine \\
\hline Simpson et al., 1992 & CRDQ & 14 & W & ja & ja & keine \\
\hline Goldstein et al., 1994 & CRDQ & 45 & W & ja & ja & keine \\
\hline Wijkstra et al., 1994; 1995 & CRDQ & 30 & W & ja & ja & 24 \\
\hline Ries et al., 1995 & QWB & 57 & INF & ja & ja & $6 / 8 / 24 / 48 / 72$ \\
\hline Watson et al., 1997 & SGRQ & 29 & W & ja & ja & keine \\
\hline Cambach et al., 1997 & CRDQ & 15 & W & ja & ja & 12 \\
\hline Büchi et al., 1997 & FLZ & 32 & nein & ja & - & 52 \\
\hline Rooyackers et al., 1997 & CRDQ & 24 & nein & ja & - & keine \\
\hline Bendstrup et al., 1997 & CRDQ, YQLQ & 16 & W & ja & ja & 12 \\
\hline Perrin et al., 1997 & SGRQ, NHP & 14 & nein & ja & - & 24 \\
\hline Fuchs-Climent et al., 1999 & NHP & 32 & nein & ja & - & keine \\
\hline Guyatt et al., 1999 & CRDQ, SIP, QWB & 38 & W & ja & ja & keine \\
\hline Gallefoss et al., 1999 & SGRO & 31 & W & nein & ja & 52 \\
\hline
\end{tabular}

SF-36 = Medical Outcomes Study Short Form [16]; QWB= Quality of Well Being Scale [7,22]; CRDQ= Chronic Respiratory Disease Questionnaire [47]; SGRQ = St. George's Respiratory Disease Questionnaire [31]; NHP= Nottingham Health Profile [57]; SIP=Sickness Impact Profile [14,40]; YQLQ= York Quality of Life Questionnaire [27]; FLZ = Fragebogen zur Lebenszufriedenheit [54]; W = Warte-Kontrollgruppe; INF = Kontrollgruppe mit unspezifischer Information über Erkrankung;

\# Abstand zur Beendigung der Maßnahme in Wochen

Corden u. Mitarb. [34] stammt eine Längsschnittstudie, in der die Wirksamkeit eines Home-Verneblers auf die HRQOL von 93 Patienten mit COPD innerhalb einer Studienperiode von vier Wochen untersucht wurde. Vor allem stand die mögliche Bedeutung der Compliance mit dem selbständig zu Hause angewendeten Vernebler im Mittelpunkt des Interesses. Zur Erfassung der HRQOL diente das SGRQ. Im Durchschnitt zeigte sich ein statistisch nicht signifikanter Trend in Richtung einer Verschlechterung der HRQOL, wobei Patienten mit einer geringeren Compliance zur Nachuntersuchung einer schlechtere HRQOL in den Bereichen der Symptomatik (SGRQ), der Symptombelastung (SGRQ) und des Gesamtwertes im SGRQ aufwies [34]. Atkins u. Mitarb. [35] prüften die Wirksamkeit unterschiedlicher psychologischer Interventionen auf die HRQOL von COPD-Patienten. Hierfür wurden fünf Gruppen von Patienten gebildet, die entweder nur Verhaltensmodifikation, nur kognitive Umstrukturierung oder eine Kombination zwischen beiden im Sinne einer kognitiven Verhaltensmodifikation erhielten. Die beiden Kontrollgruppen in der Studie von Atkins u. Mitarb. [35] bestanden aus einer Wartegruppe und einer Gruppe von Patienten, die nur eine Scheinbehandlung erhielten. Die HRQOL wurde in einem Interview mittels der Quality of Well Being Scale (QWB) $[36,37]$ erhoben. Im Vergleich zu den Kontrollgruppen fanden sich deutliche Verbesserungen in der HRQOL in allen Behandlungsgruppen, wobei der Effekt in der Gruppe der Patienten, die eine kognitive Verhaltensmodifikation erhalten hatten, am stärksten ausgeprägt war.

\section{Die Wirksamkeit von integrierten Rehabilitationsprogrammen auf die HRQOL von Patienten mit COPD}

Unabhängig davon, ob sie stationär oder ambulant durchgeführt werden, bestehen integrierte Rehabilitationsprogramme aus mehreren Bausteinen, die in der Regel Pharmakotherapie, Informationsvermittlung, Training der Atemmus- kulatur, Atemübungen, Ausdauertraining, Entspannungsübungen und verschiedene psychologische Interventionen umfassen. Zwischen den einzelnen Einrichtungen, die in der Rehabilitation tätig sind, bestehen jedoch hinsichtlich der Zusammensetzung der Programme erhebliche Unterschiede. Theoretische oder konzeptuelle Modelle für die Programmgestaltung und die Kombination der Einzelmaßnahmen einschließlich von Indikationsregeln sind bisher noch selten, zumal entsprechende Forschungsergebnisse fehlen. Tab. 2 u. 3 geben einen Überblick über Design, Methodik und Ergebnisse der bisher publizierten Studien, in denen die HRQOL zur Bewertung des Erfolgs von integrierten Rehabilitationsprogrammen diente.

Wijkstra u. Mitarb. [38] fanden bei 28 Patienten mit schwerer COPD eine signifikante Verbesserung der Anstrengungstoleranz und der HRQOL durch eine intensive Heimrehabilitation. In den Lungenfunktionsparametern blieb die Interventionsgruppe stabil, während in der Kontrollgruppe eine signifikante Verschlechterung auftrat. Der positive Effekt auf die HRQOL war demnach unabhängig von der Lungenfunktion [38]. In einer Katamnesestudie sechs Monate später konnten Wijkstra u. Mitarb. [39] zeigen, dass diejenigen Patienten, die nach Beendigung der häuslichen Rehabilitation monatlich einmal Physiotherapie zu Hause erhielten, eine signifikant bessere HRQOL im Vergleich zu denjenigen Patienten aufwiesen, die keine häusliche Physiotherapie erhalten hatten (vgl. Tab. 2 u. 3).

Büchi u. Mitarb. [40] untersuchten 32 Patienten mit COPD zu Beginn einer stationären Rehabilitation, zum Zeitpunkt der Entlassung und ein Jahr später. Die HRQOL wurde als Lebenszufriedenheit mit dem Fragebogen zur Erfassung der Lebenszufriedenheit (FLZ) [41] erhoben (vgl. Tab. 2 u. 3). Der FEV die Dyspnoe und die Lebenszufriedenheit waren zum Entlassungszeitpunkt signifikant verbessert, um sich bis zur Katam- 
Tab. 3 Effektivität von Rehamaßnahmen auf die HRQOL von COPD-Patienten

\begin{tabular}{|c|c|c|c|c|c|c|}
\hline Autoren & Setting & Intervention(en) & Dauer $\$$ & $\begin{array}{l}\text { HRQOL } \\
\text { prä-post }\end{array}$ & $\begin{array}{l}\text { HRQOL } \\
\text { Kontrolle* }\end{array}$ & $\begin{array}{l}\text { HRQOL } \\
\text { Katamnese }\end{array}$ \\
\hline Emery et al., 1991 & AMB & MOR, AUS, INF, PSY & 4 & ++ & \# & $\#$ \\
\hline Simpson et al., 1992 & AMB & MOR, MUR & 8 & + & + & $\#$ \\
\hline Goldstein et al., 1994 & STAT/AMB & MOR, MUR, AUS, INF, PSY & 8 & \# & ++ & \# \\
\hline Wijkstra et al., 1994; 1995 & HAU & MOR, MUR, AUS, INF, PSY, AT & 12 & ++ & ++ & ++ \\
\hline Ries et al., 1995 & $\mathrm{AMB}$ & AUS, INF, PSY, AT & 8 & $\mathrm{O}$ & $\mathrm{O}$ & $\mathrm{O}$ \\
\hline Watson et al., 1997 & HAU & SM & 24 & $\mathrm{O}$ & $\mathrm{O}$ & \# \\
\hline Cambach et al., 1997 & AMB & AUS, AT, INF & 12 & ++ & ++ & ++ \\
\hline Büchi et al., 1997 & STAT & AT, INF, PSY & 4 & + & $\#$ & $\mathrm{O}$ \\
\hline Rooyackers et al., 1997 & STAT & MOR, MUR, AUS & 10 & + & + & $\mathrm{O}$ \\
\hline Benstrup et al., 1997 & AMB & AUS, INF, RAU & 12 & $\mathrm{O}$ & + & + \\
\hline Perrin et al., 1997 & STAT/HAU & NIÜ & 24 & + & $\#$ & $\#$ \\
\hline Fuchs-Climent et al., 1999 & STAT & AUS, PHY, INF, PSY & 3 & + & $\#$ & \# \\
\hline Guyatt et al., 1999 & STAT/AMB & MOR, MUR, AUS, INF, PSY & 8 & + & + & $\#$ \\
\hline Gallefoss et al., 1999 & AMB & INF, SM & 1 & $\mathrm{O}$ & $\mathrm{O}$ & \# \\
\hline
\end{tabular}

INF = Informationsvermittlung; ENT = Entspannungstraining; PHY = physikalische Therapie; AUS = Ausdauertraining; RAU = Raucherentwöhnung; BT = Beschäftigungstherapie; MOR = Training Muskulatur oberer Rumpf; MUR = Training Muskulatur unterer Rumpf; AT = Atemtraining; BEW = Bewegungstherapie; SM = Selbstmanagement; PSY=Psychologische Interventionen wie z.B. Stressbewältigungstraining, Förderung der Krankheitsverarbeitung, Psychotherapie oder Entspannungstraining; NIÜ=nasale intermittierende Überdruckbeatmung; $\$=$ Wochen; ${ }^{*}=$ Vergleich mit der Kontrollgruppe; \#=keine Daten vorhanden; $\mathrm{HAU}=$ zuhause; STAT = stationär; AMB = ambulant; $++=$ deutlich besser $(p<0,001) ;+=$ besser $(p<0,05)$; $0=$ unverändert

nese jedoch wieder zu verschlechtern. Ausschließlich beim $\mathrm{FEV}_{1}$ blieb eine signifikante Steigerung im Vergleich zum Ausgangsbefund erhalten, während sich die Lebenszufriedenheit und die Dyspnoe wieder bis auf das Ausgangsniveau verschlechterten. Royackers u. Mitarb. [42] fanden eine signifikante Verbesserung der HRQOL unabhängig davon, ob die Patienten zusätzlich Sauerstoff während des Leistungstrainings erhalten hatten oder weiter Raumluft atmeten (vgl.

Tab. 4 Häufigkeit der Verwendung einzelner Methoden zur Erfassung der HRQOL zur Evaluation von Rehabilitationsmaßnahmen bei Patienten mit COPD

\begin{tabular}{llll}
\hline & Methode HRQOL & $\mathrm{n}$ & $\%$ \\
\hline krankheitsübergreifend & SIP+ & 4 & 12,9 \\
& QWB & 3 & 9,6 \\
& NHP & 2 & 6,4 \\
& SF-36 & 2 & 6,4 \\
\multirow{5}{*}{ krankheitsspezifisch } & FLZ & 1 & 3,2 \\
& CRDQ & 9 & 29,0 \\
& SGRQ & 5 & 14,0 \\
& SIP, QWB & 1 & 3,2 \\
& SF-36, SGRQ & 1 & 3,2 \\
& NHP, SGRQ & 1 & 3,2 \\
& YQLQ, CRDQ & 1 & 3,2 \\
& NHP, SF-36, CRDQ & 1 & 3,2 \\
\hline
\end{tabular}

+ einschließlich der deutschen Version der Sickness Impact Profiles (SIP); SF$36=$ Medical Outcomes Study Short Form [16]; QWB = Quality of Well Being Scale [36,37]; CRDQ = Chronic Respiratory Disease Questionnaire [27]; SGRQ = St. George's Respiratory Disease Questionnaire [15]; NHP = Nottingham Health Profile [13]; SIP = Sickness Impact Profile [19, 54]; YQLQ = York Quality of Life Questionnaire [58]; FLZ = Fragebogen zur Lebenszufriedenheit [41]
Tab. 2 u. 3). In der Studie von Goldstein u. Mitarb. [43] erhielten 89 Patienten mit schwerer COPD 8 Wochen lang eine stationäre Rehabilitation und wurden anschließend noch 16 Wochen ambulant betreut. Nach 24 Wochen wiesen die Patienten eine signifikant gebesserte HRQOL in den Bereichen „Mastery“, „Dyspnoe“ und „Emotionale Funktion“ auf. Darüber hinaus ging die Dyspnoe zurück und die Anstrengungstoleranz (Fahrradergometerleistung und Gehstrecke) hatte sich substanziell verbessert (Tab. 3 ).

Cambach u. Mitarb. [44] ließen Patienten mit Asthma oder COPD an einem gemeinsamen Rehabilitationsprogramm teilnehmen, wobei die Ergebnisse für die COPD-Patienten gesondert ausgewertet wurden. Eine Besonderheit der Studie von Cambach u. Mitarb. [44] liegt darin, dass die Kontrollgruppe als Wartegruppe in einem Cross-over-Design nach drei Monaten ebenfalls denselben Rehabilitationsmaßnahmen unterzogen wurde (vgl. Tab. 3). Es zeigten sich dann auch in der Wartekontrollgruppe signifikante Verbesserungen (vgl. Tab. 4). Es fanden sich jedoch keine substanziellen Zusammenhänge zwischen der HRQOL und der Anstrengungstoleranz sowie zwischen Verbesserungen in der Anstrengungstoleranz und der HRQOL [44].

\section{Diskussion}

\section{Gründe für den Ausschluss einzelner Studien}

Im Unterschied zu der Metaanalyse von Lacasse u. Mitarb. [11] wurde in der vorliegenden Übersicht keine erneute Datenauswertung vorgenommen. Trotz relativ „weicher“ Einschlusskriterien mussten einige Studien ausgeschlossen werden, in denen die HRQOL methodisch oder konzeptuell unbefriedigend erhoben worden war. Vergeret u. Mitarb. [29] untersuchten mittels eines adhoc-Fragebogens zur Erfassung 
der Alltagsaktivitäten die Effekte verschiedener Anwendungsformen von LTO. Das Studiendesign umfasste jedoch nur einen Messzeitpunkt und die Methodik entsprach in keiner Weise den Anforderungen an die Messung der HRQOL. In der Studie von Strijbos u. Mitarb. [45] war die HRQOL nur mittels eines Adhoc-Interviews erhoben worden. Darüber hinaus gab es auch keine echte Randomisierung [45]. Die Studie von Güell [46], die eine anhaltende Verbesserung der HRQOL nach einem intensiven Training zeigte, konnte nicht berücksichtigt werden, da sie bisher nur als Abstract vorliegt. Daher fehlen wichtige Informationen zu Methodik u. Ergebnissen. Auch in der Arbeit von Guyatt und Mitarb. [47] fehlen Informationen zu Stichprobenmerkmalen, Rehabilitationsmaßnahmen und Nachuntersuchung. Guyatt u. Mitarb. [47] zielten jedoch nicht auf die Evaluation eines Rehabilitationsprogrammes ab, sondern untersuchten die Eignung unterschiedlicher Methoden zur Erfassung der HRQOL bei Patienten mit COPD.

\section{Studien mit eingeschränkter Aussagekraft}

Die von Cambach u. Mitarb. [44] praktizierte Anwendung von identischen Rehabilitationsmaßnahmen bei Patienten mit Asthma und COPD ist problematisch, da sich beide Krankheitsbilder stark voneinander unterscheiden [2]. Darüber hinaus sind die Interventions- $(n=14)$ und die Kontrollgruppe $(\mathrm{n}=8)$ für generalisierbare Schlussfolgerungen viel zu klein, auch wenn das in der Studie von Cambach u. Mitarb. [44] verwendete Cross-over-Design besonders aussagekräftig ist. Emery und Mitarb. [48] fanden zwar in allen Subskalen des QWB eine signifikante Verbesserung der HRQOL vor und nach einer ambulanten Rehabilitation, die Stabilität des Erfolgs ist jedoch aufgrund der fehlenden Katamneseuntersuchung nicht belegt. Darüber hinaus haben Emery u. Mitarb. [48] ihre Ergebnisse nicht gegenüber einem Effekt unspezifischer Betreuungsmaßnahmen abgesichert. Die Stärke der Studie von Goldstein u. Mitarb. [43] besteht in einem sorgfältig kontrollierten, randomisierten Design, jedoch fehlt eine Nachuntersuchung zur Klärung der Stabilität der erreichten Fortschritte [42]. Gallefoss u. Mitarb. [49] führten in der Kontrollgruppe weder unspezifische noch Pseudointerventionen durch, was die Aussagekraft der Ergebnisse vermindert. Mit erheblichen Problemen ist insbesondere die Studie von Watson u. Mitarb. [50] belastet, da die Interventionsgruppe im Vergleich zur Kontrollgruppe zusätzlich noch Antibiotika und Prednison erhielt. Es fand sich zwar in der Behandlungsgruppe eine signifikant bessere HRQOL, jedoch kann ein jahreszeitlicher Effekt nicht ausgeschlossen werden, da die Studie im Spätherbst begann und im Hochsommer beendet worden war. Ries u. Mitarb. [6] fanden in einer sehr gut kontrollierten Studie mit einer Interventions- und einer Kontrollgruppe, die nur eine Schulung erhalten hatte, keinen Unterschied in der HRQOL (gemessen mittels des QWB) zwischen den beiden Gruppen, obwohl sich die Lungenfunktion in der Behandlungsgruppe deutlich gebessert hatte. Stattdessen wurde im Verlauf der Katamneseuntersuchungen über 72 Monate eine kontinuierliche Verschlechterung der HRQOL beobachtet [6]. Dieses Ergebnis ist jedoch darauf zurückzuführen, dass im QWB die verstorbenen Patienten einen Lebensqualitäts-Score von 0 erhalten, so dass sich zwangsläufig die durchschnittliche HRQOL mit zunehmender Anzahl verstorbener Patienten verschlechtert.

\section{Gesundheitsbezogene Lebensqualität (HRQOL) und Lungenfunktion als Kriterien zur Bewertung des Erfolgs pneumologischer Rehabilitation}

Es ist mittlerweile allgemein akzeptiert, dass die gesundheitsbezogene Lebensqualität (HRQOL) ein zusätzliches, von der Lungenfunktion relativ unabhängiges Kriterium zur Bewertung des Erfolges der Rehabilitation von Patienten mit COPD bildet $[5,51]$. Auch bei krankheitsspezifischen Verfahren ist dieser Zusammenhang nicht stärker ausgeprägt. Daher ist davon auszugehen, dass die einzelnen Interventionen in der pneumologischen Rehabilitation weitgehend unabhängig auf Lungenfunktion, Dyspnoe und HRQOL wirken. Dies zeigte sich auch in zwei Studien, in denen sich die HRQOL während der Rehabilitation substanziell verbesserte, die Lungenfunktion aber unverändert blieb $[38,52]$. So fanden Fuchs-Climent u. Mitarb. [52] keinen Zusammenhang zwischen Verbesserungen der HRQOL und Veränderungen der Lungenfunktion. Ebenso wurde mehrfach eine Diskrepanz zwischen der Verbesserung der Lungenfunktion (Blutgase, Dyspnoe, Anstrengungstoleranz) und einer unverändert beeinträchtigten HRQOL beschrieben [12,49]. Daher sollte die weitere Forschung klären, welche Maßnahmen im Rahmen der Rehabilitation die relativ stärkste Verbesserung der HRQOL bewirken, um dann gezielte Interventionen zur Verbesserung der HRQOL einsetzen zu können.

\section{Methodische Fragen zur Erhebung der HRQOL in Studien zur Evaluation von Rehabilitationsmaßnahmen bei Patienten mit COPD}

Verfahren zur Erfassung der HRQOL von Patienten mit COPD müssen hinsichtlich ihrer methodischen Gütekriterien in dieser Patientengruppe geprüft worden sein $[53,54]$. Von den bisher eingesetzten Verfahren liegen zum CRDQ, QWB, St. Georges Fragebogen (SGRQ) und dem SIP einschließlich seiner deutschen Version entsprechende Daten vor [54]. Studien, in denen bei COPD Patienten methodisch ungeprüfte Verfahren eingesetzt wurden, können nur mit Zurückhaltung interpretiert werden. Die Erhebung der Lebenszufriedenheit ist mit der Einschränkung einer unzureichenden Sensitivität verbunden $[53,54]$. Dieses Kriterium ist jedoch im Zusammenhang mit der Bewertung therapeutischer Interventionen besonders relevant. Wie die Tab. 4 zeigt, wurde in 14 (45\%) der 31 analysierten Studien ausschließlich ein krankheitsspezifisches Verfahren zur Messung der HRQOL verwendet, während in 12 (39\%) Studien ausschließlich ein generelles Verfahren eingesetzt worden war. In 4 der verbleibenden 5 (16\%) Studien, in denen jeweils mehrere Verfahren zur Messung der HRQOL dienten, wurde ein generelles und ein krankheitsspezifisches Verfahren miteinander kombiniert (Tab. 4).

Erstaunlicherweise wurde das krankheitsspezifische CRDQ mit 29\% am häufigsten eingesetzt, unter Berücksichtigung der kombinierten Anwendung mit anderen Verfahren sogar in einem guten Drittel (35,4\%) der Studien (Tab. 4). Diese Bevorzugung des CRDQ ist insofern problematisch, da das CRDQ erhebliche methodische Schwächen aufweist.

Bendstrup u. Mitarb. [55] fanden im krankheitsübergreifenden YQLQ im Gegensatz zum CRDQ keine substanzielle Verbesserung der HRQOL. Jedoch liegen bisher keine Daten zu 
den Gütekriterien des YQLQ bei COPD-Patienten vor. In der Studie von Perrin u. Mitarb. [56] zeigten sich im NHP sogar ausgeprägtere positive Effekte als im krankheitsspezifischen St. Georges Fragebogen. Die Befunde von Jones und Bosh [14] demonstrieren eindrucksvoll, wie mittels eines generellen Verfahrens unerwartete Nebenwirkungen einer Medikamentendosierung identifiziert werden können, die sich im krankheitsspezifischen SGRQ nicht niederschlugen. Die generellen Verfahren einschließlich des SIP sind also nicht weniger sensitiv für therapieinduzierte Veränderungen als die krankheitsspezifischen (vgl. Tab. 3). Auch konnte bisher keine klare methodische Überlegenheit der spezifischen Verfahren über die generellen bei Patienten mit COPD gezeigt werden. Daher ist aus methodischen und konzeptuellen Gründen, aber auch um allgemeine Folgen von Erkrankung und Behandlung besser erfassen zu können, eine kombinierte Verwendung von einem generellen und einem spezifischen Verfahren sinnvoll.

\section{Bewertung der Effektivität pneumologischer Rehabilitation von COPD Patienten}

Gegenwärtig ist weder eine abschließende Bewertung von integrierten Rehabilitationsprogrammen noch von einzelnen Interventionen bei Patienten mit COPD möglich. Aufgrund der bisher vorliegenden Daten führen einzelne Interventionen wie eine operative Lungenvolumenreduktion, pharmakologische Therapiemaßnahmen, Training der Atemmuskulatur, psychologische Interventionen wie aber auch integrierte pneumologische Rehabilitationsprogramme, die aus mehreren Bausteinen bestehen, zu einer anhaltenden Verbesserung der HRQOL $[2,10,14,17,18,20,21,31,32,35,38,39,42,51]$. Augenblicklich lässt sich keine klare Überlegenheit integrierter Programme, in denen Ausdauertraining, Stärkung der Muskulatur und psychologische Maßnahmen üblicherweise die Standardbausteine bilden, gegenüber Einzelmaßnahmen in Hinsicht auf die HRQOL ableiten.

Hinsichtlich der andauernden Wirksamkeit stationärer und ambulanter Rehabilitationsmaßnahmen finden sich interessante Unterschiede zwischen diesen Settings. In drei von 6 Studien, wo eine Katamnese durchgeführt worden war, konnte keine andauernde Verbesserung der HRQOL erreicht werden (Tab. 3). Hierunter waren zwei Studien, in denen die Rehabilitation ausschließlich im stationären Kontext stattgefunden hatte. Dies bedeutet, dass keine der stationären Maßnahmen, von denen eine Katamnese vorliegt, einen anhaltenden positiven Effekt auf die HRQOL bewirkte. Auf der anderen Seite hatten 3 von $4 \mathrm{im}$ ambulanten oder häuslichen Kontext durchgeführte Programme einen günstigen Effekt auf die HRQOL, der auch zur Katamnese nachweisbar war (Tab. 3). Insofern ist ein Kontexteffekt des dauerhaften Rehabilitationserfolgs bei COPD-Patienten naheliegend. Unabhängig davon, inwieweit stationäre Maßnahmen überhaupt notwendig sind, müssten demnach im Anschluss an eine stationäre Rehabilitation häusliche oder ambulante Interventionen angeschlossen werden, um einen anhaltenden Erfolg zu gewährleisten.

Die Ergebnisse von Ries u. Mitarb. [6] weisen noch auf eine andere Lücke im Netz rehabilitativer Interventionen hin. Die Autoren fanden nach einer intensiven Rehabilitation einschließlich eines Verhaltenstrainings und einer sich über ein Jahr erstreckenden monatlichen Auffrischungssitzung keine
Veränderung der HRQOL [6]. Dieses unbefriedigende Ergebnis wurde darauf zurückgeführt, dass die Patienten häufig unter Depressionen litten, was aber während der Rehabilitation nur ungenügend beachtet worden war. Daher sollten in der Rehabilitation von Patienten mit COPD vermehrt auch die emotionale Befindlichkeit, das Krankheitsverhalten und unterschiedliche Aspekte der Compliance berücksichtigt werden $[10,28,40]$. Hierin wiederum besteht ein Argument für integrierte, aus mehreren Bausteinen zusammengesetzte Rehabilitationsprogramme.

\section{Methodische Anforderungen an die "optimale“ Evaluationsstudie}

Im Rahmen von Therapieerfolgsstudien bildet ein doppeltblindes, randomisiertes Untersuchungsdesign mit einer Wartegruppe und einer Pseudobehandlungsgruppe mit unspezifischen psychosozialen Maßnahmen den methodischen Standard. Ein Cross-over-Design ist zwar aufwendig, ermöglicht aber aussagekräftige Schlussfolgerungen. Das Studiendesign sollte zumindest die Erhebung der HRQOL vor der Intervention, danach und mindestens eine Nachuntersuchung der Patienten vorsehen. Auch eine genaue Definition und Beschreibung der Interventionen ist wünschenswert. Durch Training der Behandler kann gewährleistet werden, dass alle Patienten vergleichbare Interventionen erhalten. Die Verfahren zur Erfassung der HRQOL sollten bei COPD-Patienten methodisch geprüft worden sein. Hierbei ist eine Kombination zwischen einem spezifischen und generellen Verfahren wünschenswert. Nur unter Berücksichtigung dieser Anforderungen ist die kontrollierte Erfassung der kurz- wie langfristigen Effekte von Rehabilitationsmaßnahmen und die zuverlässige Beurteilung ihrer Wirksamkeit möglich.

\section{Literatur}

${ }^{1}$ Medical Research Council Working Party. Long-term domiciliary oxygen therapy in chronic hypoxemic cor pulmonale complicating bronchitis and emphysema. Lancet 1981; I: 681

2 Petty TL, Weinmann GG. Building a national strategy for the prevention and management of and research in chronic obstructive pulmonary disease. National Heart, Lung, and Blood Institute Workshop Summary. JAMA 1997; 277: 246-253

${ }^{3}$ Ström K. Survival of patients with chronic obstructive pulmonary disease receiving long-term domiciliary oxygen therapy. Am Rev Respir Dis 1993; 147: 585- 591

${ }^{4}$ Report of British Research Medical Council Working Party. Longterm domiciliary oxygen therapy in chronic hypoxic cor pulmonale complicating bronchitis and emphysema. Lancet 1981; I: $681-686$

${ }^{5}$ Bergmann KC, Fischer J, Schmitz M, Petermann F, Petro W. Die stationäre pneumologische Rehabilitation für Erwachsene: Zielsetzung - diagnostische und therapeutische Standards - Forschungsbedarf. Pneumologie 1997; 51: 523-532

${ }^{6}$ Ries AL, Kaplan RM, Limburg TM, Previtt LM. Effects of pulmonary rehabilitation on physiologic and psychosocial outcomes in patients with chronic obstructive pulmonary disease. Ann Intern Med 1995; 122: 823-832

${ }^{7}$ National Institutes of Health Workshop Summary. Pulmonary rehabilitation research. Am J Respir Crit Care Med 1994; 149: 825-833

${ }^{8}$ European Respiratory Society, Rehabilitation and Chronic Care Scientific Group. Pulmonary rehabilitation in chronic obstructive 
pulmonary disease (COPD) with recommendation for its use. Eur Respir J 1992; 5: 266-275

${ }^{9}$ Petro W. Pneumologische Prävention und Rehabilitation. Berlin, Heidelberg, New York: Springer Verlag, 1994

${ }^{10}$ Agle DP, Baum GL, Chester EH, Wendt M. Multidiscipline treatment of chronic pulmonary insufficiency. 1. Psychologic aspects of rehabilitation. Psychosom Med 1973; 35: 41 - 49

${ }^{11}$ Lacasse Y, Wong E, Guyatt GH, King D, Cook DJ, Goldstein RS. Meta-analysis of respiratory rehabilitation in chronic obstructive pulmonary disease. Lancet 1996; 348: 1115-1119

${ }^{12}$ Van Schayck CP, Dompeling E, Rutten MPMH, Folgering H, van den Boom G, van Weel C. The influence of an inhaled steroid on quality of life in patients with asthma or COPD. Chest 1995; 107: $1199-1205$

${ }^{13}$ Hunt SM, McKenna SP, McEwen J. A quantitative approach to perceived health. J Epidemiol Comm Health 1980; 34: 281 - 285

14 Jones PW, Bosh TK. Quality of life changes in COPD patients treated with Salmeterol. Am J Respir Crit Care Med 1997; 155: $1283-1289$

${ }^{15}$ Jones PW, Quirk FH, Baveystock CM. The St. Georges Respiratory Questionnaire. Respir Med 1991; 85: 25-31

${ }^{16}$ Ware JE, Sherbourne CD. A 36-item short form health survey (SF-36): I. Conceptual framework and item selection. Med Care 1992; 30: $473-483$

${ }^{17}$ Di Lorenzo G, Morici G, Drago A, Pellitteri ME, Mansueto P, Melluso M, Norrito F, Squassante L, Falsolo A. Efficacy, tolerability, and effects on quality of life of inhaled salmeterol and oral theophylline in patients with mild-to-moderate chronic obstructive pulmonary disease. Clin Therapeutics 1998; 20: 1130-1140

${ }^{18}$ Cordova F, O'Brien G, Furukawa S, Kuzma AM, Travaline J, Criner GJ. Stability of improvements in exercise performance and quality of life following bilateral lung volume reduction surgery in severe COPD. Chest 1997; 112: 907-915

${ }^{19}$ Bergner M, Bobbitt RA, Carter WB, Gilson BS. The Sickness Impact Profile: Development and final revision of a health status measure. Med Care 1981; 19: 787-805

${ }^{20}$ Moy ML, Ingenito EP, Mentzer SJ, Evans RB, Reilly JJ. Healthrelated quality of life improves following pulmonary rehabilitation and lung volume reduction surgery. Chest 1999; 115: $383-$ 389

${ }^{21}$ O'Brien GM, Furukawa S, Kuzma AM, Cordova F, Criner GJ. Improvement in lung function, exercise, and quality of life in hypercapnic COPD patients after lung volume reduction surgery. Chest 1999; 115: 75-84

22 Nocturnal Oxygen Therapy Trial Group. Continous or nocturnal oxygen therapy in hypoxemic chronic obstructive lung disease. Ann Intern Med 1980; 93: 391 - 398

${ }^{23}$ Fleney CD, Douglas NJ, Lamb D. Nocturnal hypoxemia and longterm domiciliary oxygen in blue bloater bronchitis. Chest 1980; 77: 305

${ }^{24}$ Kearly R, Block JW, Boysen PG, Lindsley S, Martin C. The effect of low flow oxygen on sleep disordered breathing and oxygen saturation. Chest 1980; 78: $682-685$

${ }^{25}$ Heaton RK, Grant I, McSweeny J, Adams KM, Petty TL. Psychological effects of continous oxygen therapy in hypoxemic chronic obstructive pulmonary disease. Arch Intern Med 1983; 143 : $1941-1947$

${ }^{26}$ Crockett AJ, Cranston JM, Moss JR, Alpers JH. Effects of long-term oxygen therapy on quality of life and survival in chronic airflow limitation. Mon Arch Chest Dis 1999; 54: 193-196

${ }^{27}$ Guyatt GH, Berman LB, Townsend M, Pugsley SO, Chambers LW. A new measure of quality of life for clinical trials in chronic lung disease. Thorax 1987; 42: 773-778

${ }^{28}$ Muir LF, Laumonier F. Living conditions of serious chronic respiratory insufficiency patients treated at home on oxygen therapy or assisted ventilation. Lung 1990; 168: 489-494
${ }^{29}$ Vergeret J, Brabilla C, Mounier L. Portable oxygen therapy: use and benefit in hypoxaemic COPD patients on long-term oxygen therapy. Eur Respir J 1989; 2: 20-25

${ }^{30}$ Fishman DB, Petty TL. Physical symptomatic and psychological improvement in patients receiving comprehensive care for chronic airway obstruction. J Chron Dis 1971; 24: 775 - 785

${ }^{31}$ Martin JG. Clinical intervention in chronic respiratory failure. Chest 1990; 97: 105-109

32 Guyatt GH, Keller J, Singer J, Halcrow S, Newhouse M. Controlled trial of respiratory muscle training in chronic airflow limitation. Thorax 1992; 47: 598-602

${ }^{33}$ Strijbos JH, Sluiter HJ, Postma DS. Objective and subjective performance indicators in COPD. Eur Respir J 1989; 2: 666-669

${ }^{34}$ Corden ZM, Bosley CM, Rees PJ, McLellan-Cochrane G. Home nebulized therapy for patients with COPD. Patient compliance to treatment and its relation to quality of life. Chest 1997; 112: $1278-1282$

${ }^{35}$ Atkins CJ, Kaplan RM, Timms RM, Reinsch S, Lofback K. Behavioral exercise programs in the management of chronic obstructive pulmonary disease. J Consult Clin Psychol 1984; 52: $591-603$

${ }^{36}$ Kaplan RM, Bush JW. Health related quality of life measurement for evaluation research and policy analysis. Health Psychol 1982; 1: $61-80$

${ }^{37}$ Kaplan RM, Atkins CJ, Timms O. Validity of a quality of wellbeing scale as an outcome measure in chronic obstructive pulmonary disease. J Chron Dis 1984; 37: 85 - 95

38 Wijkstra PJ, VanAltena R, Kraan J, Otten V, Postma DS, Koeter GH. Quality of life in patients with chronic obstructive pulmonary disease improves after rehabilitation at home. Eur Respir J 1994; 7: $269-273$

${ }^{39}$ Wijkstra PJ, TenVergert EM, VanAltena R, Postma DS, Koeter GH. Long-term benefits of rehabilitation at home on quality of life and exercise tolerance in patients with chronic obstructive pulmonary disease. Thorax 1995; 50: 824-828

${ }^{40}$ Büchi S, Villiger B, Sensky T, Schwarz F, Wolf C, Buddeberg C. Psychosocial predictors of long-term success of inpatient pulmonary rehabilitation of patients with COPD. Eur Respir J 1997; 10: $1272-1277$

${ }^{41}$ Herschbach P, Henrich G. Der Fragebogen als methodischer Zugang zur Erfassung von „Lebensqualität“ in der Onkologie. In: Schwarz R., (Hrsg.). Lebensqualität in der Onkologie. München: W. Zuckschwerdt Verlag,: 34-46

${ }^{42}$ Rooyackers JM, Dekhuizen PNR, van Herwaarden CLA, Folgering HTM. Training with supplemental oxygen in patients with COPD and hypoxemia at peak exercise. Eur Respir J 1997; 10: 1278 1284

${ }^{43}$ Goldstein RS, Gort EH, Stubbing D, Avendano MA, Guyatt GH. Randomised controlled trial of respiratory rehabilitation. Lancet 1994; 344: 1394-1397

${ }^{44}$ Cambach W, Chadwick-Straver RVM, Wagenaar RC, van Keimpema AJR, Kemper HCG. The effects of a community-based pulmonary rehabilitation programme on exercise tolerance and quality of life: a randomized controlled trial. Eur Respir J 1997; 10: $104-113$

${ }^{45}$ Strijbos JH, Postma DS, van Altena R, Gimeno F, Koeter GH. A comparison between an out-patient hospital-based pulmonary rehabilitation program and a home-care pulmonary rehabilitation program in patients with COPD: a follow-up of 18 months. Chest 1996; 109: 366-372

${ }^{46}$ Güell R, Morante F, Sangenis M, Casan P. Effects of respiratory rehabilitation on the effort capacity and on the health-related quality of life of patients with chronic obstructive pulmonary disease. Eur Respir J 1995; 8: 356

${ }^{47}$ Guyatt GH, King DR, Feeny DH, Stubbing D, Goldstein RS. Generic and specific measurement of health-related quality of life in a 
clinical trial of respiratory rehabilitation. J Clin Epidemiol 1999; 52: $187-192$

${ }^{48}$ Emery CF, Leatherman NE, Burker EJ, MacIntyre NR. Psychological outcomes of a pulmonary rehabilitation program. Chest 1991; 100: $613-617$

${ }^{49}$ Gallefoss F, Bakke PS, Kjaersgaard P. Quality of life assessment after patient education in a randomized controlled study on asthma and chronic obstructive pulmonary disease. Am J Respir Crit Care Med 1999; 159: 812 - 817

${ }^{50}$ Watson PB, Town GI, Holbrook N, Dwan C, Toop LJ, Drennan CJ. Evaluation of a self-management plan for chronic obstructive pulmonary disease. Eur Respir J 1997; 10: 1267-1271

51 Siafakas NM, Vermeire P, Pride NB, Paoletti P, Gibson J, Howard P, Yernault JC, Decramer M, Higenbottam T, Postma DS, Ress J. on behalf of the Task Force. Optimal assessment and management of chronic obstructive pulmonary disease (COPD). Eur Respir J 1995; 8: $1398-1420$

52 Fuchs-Climent D, Le Gallais D, Varray A, Desplan J, Cadopi M, Prefaut C. Quality of life and exercise tolerance in chronic obstructive pulmonary disease: effects of a short and intensive inpatient rehabilitation program. Am J Phys Med Rehabil 1999; 78: $330-335$

${ }^{53}$ Bullinger M. Gesundheitsbezogene Lebensqualität und subjektive Gesundheit. Psychother Psychosom Med Psychol 1997; 47: $76-91$

${ }^{54}$ Hütter BO, Würtemberger G. Lebensqualität bei Patienten mit chronisch-obstruktiven Lungenerkrankungen: Psychometrische Gütekriterien der deutschen Version des Sickness Impact Profiles (SIP) und erste Ergebnisse der Anwendung. Pneumologie 1997; $51: 108$ - 114

${ }^{55}$ Benstrup KE, Jensen JI, Holm S, Bengtsson B. Out-patient rehabilitation improves activities of daily living, quality of life and exercise tolerance in chronic obstructive pulmonary disease. Eur Respir J 1997; 10: 2801 - 2806

56 Perrin C, El Far Y, Vandenbos F, Tamisier R, Dumon MC, Lemoigne F, Mouroux J, Blaive B. Domiciliary nasal intermittent positive pressure ventilation in severe COPD: effects on lung function and quality of life. Eur Respir J 1997; 10: 2835-2839

${ }^{57}$ Jones PW, Baveystock CM, Littlejohns P. Relationships between general health measured with the Sickness Impact Profile and respiratory symptoms, physiological measures and respiratory symptoms, physiological measures and mood in patients with chronic airflow limitation. Am Rev Respir Dis 1989; 140: 1538 1543

${ }^{58}$ Gudex C, Kind P. The QUALY Toolkit. Discussion paper 38. York, Centre for Health Exonomics: University of York 1988, 1988

PD Dr. med. G. Würtemberger

Abtlg. für Innere Medizin, Pneumologie

Reha-Zentrum Reichshof

Zur Reha-Klinik 1

51580 Reichshof-Eckenhagen

\section{BUCHBESPRECHUNGEN}

L. S. Bickley (Hrsg.): Bates' großes Untersuchungsbuch, 3. Auflage, Deutsche Übersetzung. Georg Thieme Verlag, Stuttgart 2000, 787 S., DM 128,-. ISBN 3-13-117633-4

Anamnese und körperliche Untersuchung sind gerade im Zeitalter explodierender Medizintechnologie und gleichzeitig gedeckelter Budgets unverzichtbar, damit apparative Methoden gezielt und zum Wohle des Patienten eingesetzt werden können. Das Lehrbuch von Bates, das seit seiner Erstauflage 1974 als Klassiker auf dem amerikanischen Markt gilt, bietet alle Informationen, die man für eine vollständige klinische Statuserhebung benötigt.

Für die Untersuchung jedes Organgebietes wird zunächst eine anatomische Übersicht gegeben, die didaktisch hervorragende Abbildungen umfasst. Die Untersuchungstechniken sind teils mit Fotos, teils mit Schemazeichnungen gut verständlich illustriert und im Text ausführlich beschrieben. Besonders positiv hervorzuheben sind Hinweise wie z. B. bei der Beschreibung der Palpation axillärer Lymphknoten: „Weisen Sie den Patienten daraufhin, dass dies unangenehm sein kann" oder bei der Auskultation der Lunge: „Achten Sie darauf, dass der Patient nicht hyperventiliert und lassen Sie ihn gegebenenfalls eine Pause machen“. Pathologische Untersuchungsbefunde, Tipps und klinische Anmerkungen finden sich übersichtlich auf den breiten gelben Randstreifen in unmittelbarer Nachbarschaft zum Haupttext. Übersichtliche Tabellen runden das Werk ab. Einige Fachtermini wie „Egophonie“ oder „Pektoriloquie“ waren mir bisher nicht geläufig; dafür vermisste ich „Knisterrasseln“, „Platypnoe“, „Crepitatio indux/redux“. Sehr ausführliche Kapitel behandeln Gesprächsführung und Anamnese sowie die Erhebung des psychischen Befundes.

Das seit der letzten Auflage komplett überarbeitete Werk, das insgesamt fast 1000 klinische Fotos und instruktive Graphiken sowie ca. 110 Riesentabellen umfasst, ist überaus gut lesbar, nicht zuletzt deshalb, weil der Satzbau einfach ist, auf Medizinerlatein wann immer möglich verzichtet wird und weil der Leser sich durchgängig persönlich angesprochen fühlt. Das Buch kann uneingeschränkt empfohlen werden.

G. Kanzow, Großhansdorf 